Programmatische Kommentare und Repliken

Doris Bachmann-Medick*

\title{
Kulturwissenschaft in der Ermüdung? Anmerkungen zu einer Neuorientierung
}


Abstract: Starting from the conviction that the study of culture(s) is much broader than a philosophizing history of ideas approach (one that often retains implicit Eurocentric assumptions), this article is a plea for a reorientation of the study of culture through the demonstration of a stronger commitment to a sociological, empirical and transcultural approach in the study of culture. Instead of focusing on cultural syntheses (i.e. along the main signatures and "Zeitgeist" symptoms of epochs), my argument redirects attention to particularities, hidden dimensions, and the formation of differences, to cultural countermovements and contradictions. The article suggests a more complex and action-oriented "translational" approach. It aims to foster a critical self-reflection of the research process of the study of culture itself with regard to its analytical concepts, its societal and ethical concerns, and its fruitful convergence of disciplines.

Keywords: philosophical-historical study of culture - empirical, sociological and transcultural study of culture - transnationalization - "translational" approach - self-reflection of research processes cultural differences - cultural perceptions - analytical categories - emerging topics - concepts and concerns - management/manageability of research - „public humanities"

*Dr. Doris Bachmann-Medick: International Graduate Centre for the Study of Culture (GCSC), Justus-LiebigUniversität Gießen, Alter Steinbacher Weg 38, D-35394 Gießen, email: bachmann-medick@web.de

In Hartmut Böhmes Text (wie zuweilen auch in meinen eigenen Texten) ist eine weit verbreitete Schreibpraxis zu beobachten. Sie neigt dazu, die typische metaphorische Verwendung kulturwissenschaftlicher Begriffe überzustrapazieren, ohne sie auf empirische Bezüge hin zu konkretisieren und sie auf diese Weise klar genug vom Jargon fernzuhalten. Ist es nicht an der Zeit, solche metaphorischen Umschreibungen rückzuorientieren auf Analysekategorien, auf operationalisierbare ,Konzepte', ja auf die Herausforderungen der gesellschaftlichen Problemlagen? So könnte von den Kulturwissenschaften aus die empirische und systematische Arbeit der Disziplinen bereichert werden, grenzüberschreitend und auf globale Horizonte hin geöffnet.

Dies klingt durchaus programmatisch. Nicht jedoch - um es gleich zu betonen - für ein Einzelfach ,Kulturwissenschaft'. Ein solches steuert Hartmut Böhme an, wenn er vor allem „die programmatische Absicht auf ,Kulturwissenschaft' einlösen will" (vgl. Böhme in diesem Heft). Hinter diesem Horizont eröffnen sich jedoch viel weiterreichende programmatische Perspektiven, sobald man Kulturwissenschaft(en) pluralisiert und sie damit selbst als ein "fächerübergreifendes ,Forschungsprogramm'" (Reckwitz 2011: 1) versteht, das immer wieder zum Überdenken der eingerasteten disziplinären Schlüsselbegriffe und festgefügten historischen Periodisierungen aufruft, ja das die gewohnten Untersuchungsfelder gründlich umpflügt. Dazu reicht es allerdings nicht, die kulturwissenschaftliche Offenheit für bisher Unerhörtes, Ungesagtes, Ausgeblendetes, Nicht-Sichtbares, Marginalisiertes, bloß Alltägliches in erster Linie nur zur Erschließung neuer, ungewöhnlicher Phänomene und Themenfelder zu nutzen - wie etwa Müdigkeit und Erschöpfung (bei Böhme), Sensibilität, Langeweile, Ekel, aber auch, man staune, die Geschichte des Parkhauses oder der Sonnenbrille.

Viel weitreichender ist vielmehr das Potential neuer Analysekategorien. Gemeint sind operative, differenzierende methodische Untersuchungslinsen, die solche Themen gerade nicht in Zuständen aufgehen lässt, sondern sie auf die Vielschichtigkeit von Handlungsoptionen hin entfalten. Dadurch werden sie kulturwissenschaftlich besonders interessant. Denn der analytische Blick richtet sich hier bereits auf die Problematik unausgesprochener, aber handlungssteuernder Vorannahmen, kultureller Klassifikationen, binärer Wahrnehmungsmuster, Universalisierungsansprüche usw. Er richtet sich auch auf das Zusammen- und Gegeneinanderwirken der unterschiedlichsten, oft widersprüchlichen 
Praktiken und Wissensordnungen im jeweiligen Untersuchungs(zeit)raum, die dann gerade nicht eingeebnet werden zu komplementären Paarungen (wie Arbeit und Müdigkeit). Es ist die Dynamik der cultural turns, welche eben nicht nur neue Erkenntnisobjekte entdecken (Raum, Ritual, Bild, Performanz, Übersetzung usw.), sondern diese vielmehr zu eigenen Erkenntnismitteln ausarbeiten, schärfen und als Analysekategorien nutzbar machen - weit über die eigene Kultur und Kulturgeschichte hinaus. Nicht von vornherein mit kulturspezifischen Inhalten verknüpft, bieten sie sich für transkulturelle Forschungsfelder geradezu an. Gemeint sind hier die disziplinenübergreifenden Analysekategorien der neueren, empiriegeladenen und transkulturell geöffneten Kulturwissenschaften.

\section{Philosophische Kulturwissen- schaft - europazentriert}

Hartmut Böhmes Text folgt jedoch anderen Wegen. Gemeint sind die europäischen Bahnen einer philosophischen Kulturwissenschaft - weitgehend an die historischen Kulturwissenschaften der 1920er/1930er Jahre anknüpfend, eher weniger an die internationalisierten Kulturwissenschaften am Ende des 20. und Beginn des 21. Jahrhunderts. Und doch betont Böhme gleich zu Anfang: „Es gibt nicht ,die Kultur', sondern nur Kulturen" - und diesem Pluralisierungsansatz als grundlegendem Charakteristikum einer kulturwissenschaftlichen Herangehensweise kann man nur zustimmen. Da aber fängt das Problem allererst an: Auch in der Rede von Kulturen schwingt noch allzu oft die Vorstellung abgrenzbarer Ganzheiten oder gar Container mit, ebenso die Neigung zum Essentialisieren, wie es die neueren Kulturwissenschaften gerade vermeiden wollen. Hätte es nicht größeren Reiz, vom "Kulturellen" als einer "Arena von machtförmigen Aushandlungsprozessen" (Lentz 2009: 307) zu sprechen oder auch - wie Andreas Reckwitz dagegenhält - von Kultur als einem eher "präreflexiven Wissen", das immer schon über Praktiken wirksam wird (Reckwitz 2009: 417)?

Damit wären jedenfalls Modi des Kulturellen freigelegt, die für transkulturelle Perspektiven anschlussfähiger sind. Nicht so in Böhmes
Text, der die Kulturwissenschaft unbeirrt auf "die europäische Kultur", ja auf "die Moderne" einhegt. Dabei schmuggeln sich neben einem eurozentrischen Zuschnitt zugleich wiederum eine Singularisierung und Typisierung, ja das kulturelle Gesamtbild einer "synthetischen Kulturbeschreibung" (James Clifford) ein: Weit entfernt vom mikroskopischen Blick einer stärker empiriehaltigen kulturwissenschaftlichen Forschungspraxis, die Einzelheiten, Subjekte (nicht nur Subjekttypen), Differenzbildungen und produktive Gelenkstellen zu ihrem Ausgangspunkt nimmt, haben wir es hier mit einer Kulturwissenschaft der longue durée, der langen, zeitenüberspannenden Wellen zu tun. Wir sehen eine philosophische Kulturwissenschaft am Werk, die sich aus dem Feld der Ideengeschichte speist und die sich den langen Wellen von Themen zuwendet, welche "die Moderne" mitprägen - Nerven, Risiko, Kontingenz, Religion - auch den "Theorien ästhetischer Autonomie", wie sie Böhme aus den „Topoi des anti-urbanen Landlebens" ableitet.

\section{Empiriegeladene Kulturwissen- schaften - soziologisch konkret, transkulturell offen}

Was einer solchen philosophischen Kulturwissenschaft fehlt - und was in vielerlei Hinsicht gerade eine kulturwissenschaftliche Herangehensweise auszeichnet - ist der differenzierende Blick auf unbeleuchtete Gegenbewegungen, auf kontrastierende und widerständige Handlungen (nicht nur Stimmungen), ja auf Epochenuntergründe. Gerade solche Gegenläufigkeiten zum „Zeitgeist" fordern dazu heraus, den mainstream immer wieder zu irritieren, etwa durch eine "defamiliarization by (...) cross-cultural juxtaposition" (Marcus/ Fischer 1999: 138), wie sie die Kulturanthropologie methodisch gefordert hat, oder durch "contrapuntal reading" (Said 1993: 66), wie man es von Edward W. Said her kennt. Kein europäisches (kanonisches) Kunstwerk - so Said - kommt aus ohne sein Gegenstück in den Geschichten und Erfahrungen des Kolonialismus, die meist unthematisiert und doch deutlich erkennbar, in ihm selbst durchscheinen. Man lese nur die Romane 
von Jane Austen. "We must therefore read the great canonical texts (...) with an effort to draw out, extend, give emphasis and voice to what is silent or marginally present or ideologically represented (...) in such works" (Said 1993: 66). Doch ein solches Spannungsverhältnis von widersprüchlichen Überschneidungen sprengt scheinbar festgefügte epochale Einheiten und Begrenzungen. Erkennt man dies an, so kann man eigentlich nur noch quer zu griffig gemachten „Epochensignaturen" arbeiten.

Ganz anders jedoch, wenn man den großen - europazentrierten - Bogen einer Genealogie der Moderne nachzeichnet und dabei ein "riesiges Diskursfeld" (vgl. Böhme, ebd.) aufmacht, das ganze Epochen überspannt. Anders auch, wenn man sich auf das fixiert, was man als die Dispositionen der Moderne zu erkennen meint, z.B. auf eine allumspannende "Müdigkeit" ohne freilich die "Müdigkeit" des kapitalistischen Fabrikarbeiters dann noch zu unterscheiden von der solipsistischen „Müdigkeit" des Dichterindividuums bei Peter Handke. Mit den sozial bzw. soziologisch rückbezogenen Differenzierungen einer kulturwissenschaftlichen Analyse im Licht einer „notwendigen ,Resoziologisierung' des Kulturbegriffs" (Lentz 2009: 320) hingegen lieBen sich die Erkenntnismöglichkeiten erweitern. „Müdigkeit" würde dann nicht nur als Zustandsdiagnose festgestellt, sondern als Teil einer konkreten Praxis untersucht: als ein Reaktionsverhalten gegenüber sozial ungleichen Arbeitsverhältnissen, das schichtenspezifisch differenziert ist und je eigene Bewältigungsstrategien ausprägt. Statt einen solchen komplexen Praxiszusammenhang ideengeschichtlich zusammenzuschweißen, wäre ausdrücklich in ihn einzuhaken, auch um seine „präreflexiven" Vorformungen freizulegen.

Geht hier die philosophisch-ideengeschichtliche Kulturwissenschaft à la Böhme also differenzierend genug vor? Geschichte erscheint da doch eher als ein überspannendes Fluidum, verdichtet in "Leitmetaphern" (vgl. Böhme, ebd.). Letztlich wird der historische Prozess auf ein Äquilibrium zusammengezogen, auf ein Gleichgewicht von Dualismen und komplementären „Mentalitäten". Differenzen, Gegenläufigkeiten, Widersprüchlichkeiten, Widerständigkeiten könnten damit allzuleicht eingeebnet werden. Ist hier, zugespitzt gefragt, etwa eine Kulturwissenschaft am Werk, die selbst dabei ist zu ermüden?

\section{Wahrnehmungsdispositionen als Analysekategorien}

Eine differenzorientierte kulturwissenschaftliche Herangehensweise hingegen macht Grenzbereiche und Verschiebungen zwischen den Disziplinen - hier in Richtung der Soziologie - produktiv. So markiert sie stärker die Brüche, die Abweichungen und Missverhältnisse, um sie dann weitergehend zu analysieren: als soziale und gesellschaftliche Ungleichheiten. Am Ende der Metaphernkette des von Böhme durchaus eindrucksvoll nachgezeichneten Zeitalters der Nerven und der "modernen Müdigkeit" (vgl. Böhme, ebd.) wäre durchaus der Umschlag zu einer solchen kulturwissenschaftlichen Untersuchung vorstellbar, die mit weitaus konkreteren Analysekategorien operiert. Statt nur Leitmetaphern auf ihre kulturelle Symptomfunktion hin nachzuspüren, könnten diese auf differenzerhaltende Konzepte hin geöffnet werden, die eher auf eine kulturelle Translationsfunktion verweisen.

Auch Hartmut Böhme verwendet ja „Müdigkeit" im Modus einer Analysekategorie. Von hier aus ließe sich in Richtung auf eine empiriegeleitete kulturwissenschaftliche Perspektive weiterarbeiten - ganz bestimmt genauer, vielleicht auch dorniger als durch ihre Verschmelzung zu einer Epochensignatur "der Moderne". Dafür lieBen sich die Wahrnehmungskategorien, wie sie Böhme in seinem Text herausgearbeitet hat, wiederum nutzen und weiter ausbauen. Solche Wahrnehmungsdispositionen werden ja, wie er deutlich macht, überhaupt erst durch eine kulturwissenschaftliche Linse als Phänomene zur Geltung gebracht, die sich zu erforschen lohnen. Doch wären sie eben nicht nur als „Mentalitäten" (vgl. Böhme, ebd.) ernstzunehmen, sondern selbst (und selbstkritisch) auf ihre Kulturabhängigkeit hin zu reflektieren.

So könnte aus den Wahrnehmungsdispositionen dieser Epochensignaturen, wie sie Böhme bezeichnet, eine kulturwissenschaftliche Erkenntniseinstellung hergeleitet werden, die sich nicht mehr nur historisch selbstvergewissert, sondern die sich ausdrücklich den Gegenwarts- und Zukunftsherausforderungen aussetzt. Dies allerdings verlangt, sich auf die soziale Rückbindung solcher Wahrnehmungsdispositionen und ihrer kulturspezifischen Praktiken einzulassen. Solche 
Kategorien der Wahrnehmungsprägung bis hin zu Kulturtechniken können - wie die Umwandlungsprozesse von metaphorischen in analytische Begriffe im Feld der cultural turns zeigen - ausgebaut werden zu methodisch geschärfteren Analysekategorien, ohne diese freilich zu enthistorisieren. Man denke nur an die Kategorie der Übersetzung, die im Zuge des translational turn zunächst zu einer wuchernden Ausweitung des Übersetzungsbegriffs auf alle möglichen kulturellen und längst nicht mehr nur sprachlichen Felder geführt hat. Erst indem sie verstärkt rückgebunden wurde an empirische Fallstudien konnte sie konkreter für die Analyse einzelner Übersetzungsschritte in sozialen Interaktionszusammenhängen oder gesellschaftlichen Transformationsprozessen genutzt werden. So bringen translatorische Analysen in den empirischen Feldern von Mission und Konversion, von Diplomatie, Gesellschaftstransformation, aber auch von Menschenrechtsdiskursen neue Erkenntnisse über die genauen Abläufen von Übergängen. ${ }^{1}$ Man kann sogar noch weiter gehen: Kulturwissenschaftliche Ansätze überhaupt zeichnen sich nicht selten durch eine Aufmerksamkeit auf Übersetzungsschritte aus, auf analytische Zergliederungen, die eben nicht vorschnell synthetisieren - um so etwa Transformationsprozesse herauszuarbeiten und die veränderungsrelevanten Gelenkstellen und Anschlussstellen dafür genauer benennen zu können. Denn Kulturwissenschaften, wie Böhme eher am Rande zugesteht, beschäftigen sich ja auch mit "engbegrenzten Objektfeldern in chronotopischer Verdichtung" (vgl. Böhme, ebd.). Aber auch hier wäre die Verdichtung wiederum zu entflechten, etwa durch den mikroskopischen Blick eines translatorischen Zugriffs, der Synthesebegriffe und Globalperspektiven aufbricht.

Eine solche empirische Konkretisierung der Begrifflichkeit, wie sie am Beispiel von Übersetzung als Analysekategorie vorgeführt werden kann, ist Bestandteil einer wichtigen und umfassenderen kulturwissenschaftlichen Praxis: der Selbstreflexion der jeweiligen (auch der eigenen) Wissenschaftsbedingungen. Nicht nur die kriti-

\footnotetext{
1 Aus einer mittlerweile Vielzahl von neueren Fallbeispielen sei auf das Themenheft "Übersetzungen" der Zeitschrift "Geschichte und Gesellschaft" verwiesen (2012), darin u.a. Doris Bachmann-Medick (2012a): Menschenrechte als Übersetzungsproblem, 331-359.
}

sche Einbindung in die jeweils zugrundeliegende kulturspezifische Erkenntnisordnung ist damit gemeint, sondern auch ein Überdenken der eingefahrenen Forschungspraxis (nicht zu vergessen die zunehmende, uns allen bekannte und angesichts des Publikationsdrucks fast schon unvermeidliche Mehrfachverwertung der eigenen Texte oder Textpassagen - auch Böhmes Impulstext enthält erhebliche Abschnitte, die bereits in seinem Aufsatz "Das Gefühl der Schwere" (Böhme 2015) veröffentlicht wurden). Die neueren Ansätze der "public humanities" 2 haben zudem die Aufmerksamkeit gelenkt auf das Problem der Vermittlung der geistes- und kulturwissenschaftlichen Forschungsergebnisse in die gesellschaftliche Öffentlichkeit hinein. Darüber hinaus ist eine noch tiefer reichende Selbstreflexion der eigenen Untersuchungsbegriffe zur entscheidenden Forderung einer kulturwissenschaftlichen Forschungspraxis geworden. Begriffe wie Arbeit, Freiheit, Natur, Kunst, Religion sind ja keineswegs allgemeingültige, universalisierbare Ausgangspunkte der Forschung - eine Einsicht, die auch die europäische Begriffsgeschichte, auf der die Ideengeschichte ja beruht, weiterhin fragwürdig macht.

Es gilt also noch vor jeglicher Epochenbeschreibung anzusetzen - eben bei den Begriffen und Erkenntnisvoraussetzungen selbst, die ja als solche bereits kulturspezifisch geprägt sind. Hier muss ich - wenngleich auch ich dies schon an anderen Orten getan habe (Bachmann-Medick 2012: 34f.) - noch einmal auf Dipesh Chakrabartys höchst anregendes Konzept der "cross-categorical translation" verweisen, die mit jeglicher "cross-cultural translation" einhergehen sollte (Chakrabarty 2000: 83ff.). Anzusetzen ist hiernach bereits an den keineswegs selbstverständlichen Kategorien, an den Konzepten selbst Konzepte verstanden im Sinne von Mieke Bal als Mini-Theorien, die sich zwischen den Disziplinen, ja auch durch unterschiedliche kulturelle Kontexte hindurch bewegen, die dabei als "travelling concepts" (Bal 2002) wirken. Statt bei Metaphern stehenzubleiben oder bei den europäisch geprägten Begriffen, wäre vielmehr die verwendete Begrifflichkeit auf solche "travelling concepts" hin

2 Siehe zu diesem hierzulande noch wenig bekannten Diskurs: u.a. Brooks/Jewett 2014; Cooper 2014; Nussbaum 2010. 
zu befragen - nicht zuletzt, um sie für transkulturelle Kulturforschung einsetzbar zu machen.

Wo finden sich in Hartmut Böhmes Text "travelling concepts"? Wie steht es da beispielsweise mit der ästhetischen "Autonomie"? Ist dies nicht auch eine profund europäische Vorstellung, die im weltliterarischen Horizont längst nicht mehr universalisierbar ist, die keineswegs unumstritten "reist", die vielmehr im Sinne Chakrabartys "provinzialisiert" werden müsste? Mit dem Gebrauch solcher kulturspezifisch verengten Untersuchungsbegriffe wird eine "synthetische Kulturbeschreibung", wie sie James Clifford schon in den 1980er Jahren im Zuge der Writing Culture Debatte kritisiert hatte, auf jeden Fall eher noch bekräftigt als aufgebrochen. Verengt werden damit mögliche Anschlüsse an andere Modernen in der Weltgesellschaft. Zusammengezogen zu einem neuen „Bild" der europäischen Moderne - von Müdigkeit über ästhetische Autonomie bis hin zu Risiko, Unsicherheit, Kontingenz - schließt sich damit auch eine Kulturwissenschaft, die dieses Bild produziert, geradezu selbst ab.

\section{Dynamik der Kulturwissen- schaften - Emerging Topics, Gesellschaftsbezüge, Disziplinenüberlappungen}

Käme es nicht aber darauf an, die Dynamik der Kulturwissenschaften über die Disziplinen- und Kulturgrenzen hinaus weiterzutreiben? Geschieht dies durch die zunehmenden Verschiebungen, Resemantisierungen und produktiven Kontextwechsel, wie sie gegenwärtig am Werk sind? Hartmut Böhme hat solche faszinierenden Verschiebungen des Religiösen in Form "flottierender Energien" (vgl. Böhme, ebd.) in die Sphäre des Sports - kennzeichnend für heutige postsäkulare Gesellschaften - eindrucksvoll vor Augen geführt. Geschieht dies - wissenschaftsorganisatorisch gesehen - weiterhin durch turns? Oder eher durch die Entwicklung von studies quer zu den etablierten Fächern (Moebius 2012: 7-12, bes. 11)? Vielleicht sogar durch einen ,turn' der Kulturwissenschaften selbst, durch ihre Abkehr von kulturwissenschaftlicher Beliebigkeit, in die ein zu sorgloser Umgang mit turns führen könnte?
Was sich in der Tat in jüngster Zeit abzeichnet, ist eine deutlichere Hinwendung zu ethischen Fragen, die Anerkennung kulturwissenschaftlicher Verantwortlichkeiten sowie nicht zuletzt eine stärkere Verpflichtung auf Gesellschaftsbezüge. Dies bedeutet auch, sich mit aufkommenden Forschungsfeldern, mit emerging topics auseinanderzusetzen und diese in ihrer gesellschaftlichen Brisanz vonseiten der Kulturwissenschaften ernstzunehmen. In dieser Hinsicht spricht man in den gegenwärtigen, zukunftsorientierten Kulturwissenschaften nun nicht mehr nur von travelling concepts, sondern von einer Weiterentfaltung solcher Konzepte hin zu concerns angesichts der dringenden Gesellschafts-, ja Weltprobleme wie z.B. Religionskonflikten, Klimawandel, Altern, Mobilität, Migration usw. Und so reicht eben nicht mehr nur die Rückwendung zu Diagnosen "der Moderne", wie kulturwissenschaftlich diese auch immer angelegt sein mögen: mit Blick auf Müdigkeitsphänomene, Risikoverhältnisse oder Kontingenzunahme.

Besonders produktiv ist in diesem Zusammenhang vielmehr die Ausdehnung der Kulturwissenschaften in Grenzbereiche und Überlappungsfelder hinein. Hier lohnt es sich weiterzuarbeiten. Denn Verschiebungen können auch hier höchst produktiv sein - Verschiebungen etwa im Gefüge der Disziplinen selbst. Ein solches Aufeinanderzubewegen der Fächer wäre bei einem Einzelfach Kulturwissenschaft nur schwer möglich. Denn es ist ja gerade das fächerübergreifende systematische Anregungspotential der Kulturwissenschaften, das von den unterschiedlichsten Fächern aufgegriffen und auf ihre Weise verarbeitet wird. Ein Beispiel für ein solches Aufeinanderzubewegen wäre die kulturwissenschaftliche Neuorientierung, die neuerdings von den management und organizational studies ausgeht - durch deren Selbstreflexion im Licht der cultural turns (Bachmann-Medick 2016). Hier ließe sich anschließen an Böhmes entscheidende Hervorhebung, dass Nutzen in kulturwissenschaftlicher Sicht ein strikt ökonomisches Verständnis überwindet, indem es - à la Bourdieu - auch das symbolische Kapital umfasst (vgl. Böhme, ebd.). Aber eben auch für die kulturwissenschaftliche Forschungspraxis selbst kommt die ökonomische Dimension stärker denn je in den Blick. Hier könnte etwa das kritische Umdenken der Manager-Autorität im Feld der critical management studies kon- 
krete Vorschläge an die Hand geben: Praktiken wie Vernetzung, Konnektivität, transnationale Öffnung wären stärker in den Vordergrund zu rücken als herkömmliche, individuumbasierte Strukturen von Steuerung und Management. Dies könnte nicht zuletzt auch manch kritische Fragen anregen: Ob und wie laufen eigentlich im Bereich der (Kultur-)Wissenschaften selbst Steuerungs- und Managementprozesse $a b$ ? Wie werden etwa Forschungsstrategien lanciert und auf den Weg gebracht, welche Markt- und Effizienzorientierungen sind am Werk und wie bilden sich dennoch - durchaus unabhängig von einer inhaltlichen Programmatik der Kulturwissenschaften immer wieder überraschende Konjunkturen von Theorien heraus? Wenn Lawrence Grossberg mit seinem Aufruf: "rescuing economies from economists" (Grossberg 2010: 101) 3 ausdrücklich auf die anglo-amerikanischen cultural studies und ihre Pluralisierungsanstöße verweist, dann könnte ein entsprechender Aufruf wiederum auch die Kulturwissenschaftler_innen hierzulande aufrütteln: durch mutigere Grenzüberschreitungen in scheinbar entfernte Disziplinen hinein könnten auch die Kulturwissenschaften vor sich selbst gerettet werden - vor ihrer Ermüdung, aber auch vor ihrer selbstgenügsamen Selbstübertreibung.

\section{Literaturverzeichnis}

Bachmann-Medick, Doris (2012): Translation - A Concept and Model for the Study of Culture. In: Neumann, Birgit/Nünning, Ansgar (Hgg.): Travelling Concepts for the Study of Culture. Berlin/Boston: De Gruyter, S. 23-43.

Bachmann-Medick, Doris (2012a): Menschenrechte als Übersetzungsproblem. In: Geschichte und Gesellschaft 38.2, Themenheft "Übersetzungen", hg. von Simone Lässig, S. 331-359.

Bachmann-Medick (2016), Doris: Cultural Turns - A Matter of Management? In: Küpers, Wendelin/ Sonnenburg, Stephan/Zierold, Martin (Hgg.): ReThinking Management. Wiesbaden/New York: Springer VS [im Erscheinen].

Bal, Mieke (2002): Travelling Concepts in the Humanities: A Rough Guide. Toronto et al.: The University of Toronto Press.

Böhme, Hartmut (2015): Das Gefühl der Schwere. Historische und phänomenologische Ansichten der

3 Vgl. in diesem Sinn Grossberg (2010): 168: "economies are too important to be left to economists."
Müdigkeit, Erschöpfung und verwandter Emotionen. In: figurationen 1, S. 26-49.

Brooks, Peter/Jewett, Hilary (Hgg.) (2014): The Humanities and Public Life. New York: Fordham University Press.

Chakrabarty, Dipesh (2000): Provincializing Europe: Postcolonial Thought and Historical Difference. Princeton/Oxford: Princeton University Press.

Cooper, David D. (2014): Learning in the Plural: Essays on the Humanities and Public Life. East Lansing: Michigan State University Press.

Grossberg, Lawrence (2010): Cultural Studies in the Future Tense. Durham, MA/London: Duke University Press.

Lentz, Carola (2009): Der Kampf um die Kultur. Zur Ent- und Re-Soziologisierung eines ethnologischen Konzepts. In: Soziale Welt 60, S. 305-324.

Marcus, George E./ Fischer, Michael M. J. (1999): Anthropology as Cultural Critique: An Experimental Moment in the Human Sciences. 2. Aufl. Chicago/ London: University of Chicago Press.

Moebius, Stephan (2012): Kulturforschungen der Gegenwart - die Studies. Einleitung. In: ders.: Kultur. Von den Cultural Studies bis zu den Visual Studies. Eine Einführung. Bielefeld: transcript.

Nussbaum, Martha C. (2010): Not for Profit: Why Democracy Needs the Humanities. Princeton/Oxford: Princeton University Press.

Reckwitz, Andreas (2009): (Ent-)Kulturalisierungen und (Ent-)Soziologisierungen. Das Soziale, das Kulturelle und die Macht. Ein Kommentar zu Carola Lentz: „Der Kampf um die Kultur: Zur Ent- und Re-Soziologisierung eines ethnologischen Konzepts". In: Soziale Welt 60, S. 411-418.

Reckwitz, Andreas (2011): Die Kontingenzperspektive der ,Kultur'. Kulturbegriffe, Kulturtheorien und das kulturwissenschaftliche Forschungsprogramm. In: Jaeger, Friedrich/Liebsch, Burkhart (Hgg.): Handbuch der Kulturwissenschaften, Bd. 1. Stuttgart/Weimar: Metzler, S. 1-13.

Said, Edward W. (1993): Culture and Imperialism. New York: Alfred A. Knopf. 